could only be counted at the apex. Everything taken by the mouth was vomited, so rectal feeding was resorted to and nursing was stopped. The same condition continued for two days, nothing seeming to have any effect on the heart's action, except when strychnia was given, and then the rapidity was increased.

At this time I was called out of town and was gone two days, leaving her in charge of Dr. C. H. Hare. During the second day of his attendance he noticed that the apex beat was below the fifth interspace and outside of the mammary line.

On my return I found her very much agitated because her friends were urging the calling in consultation a physician who had seen her before her marriage, but whom she disliked. I asked that Dr. H. Jackson be called. He agreed in the diagnosis that dilatation was beginning, and advised raising the head, keeping an ice bag over the cardiac region and giving $10 \mathrm{~mm}$. of fat free digitalis every two hours. The raised position made her so uncomfortable that it was abandoned. She was nauseated after the second dose of digitalis so after that it was given by rectum. Codeia from $\frac{1}{8}$ to $\frac{1}{2}$ gr. was given as needed to insure sleep.

The next day she seemed better, although the heart rate was still 180 . She objected so much to the ice bag that that was omitted. During the next five days no change was noted. At the end of that time coarse râles were found at the base of the right lung. That afternoon Dr. Jackson saw her again with me, and found a pleuritic friction at the right base, with an enlarged and pulsating liver. The next morning I was informed by her husband that my services were no longer needed as he had found a doctor who was a heart specialist and who said he could cure the patient in thirty-six hours.

The two nurses who had been in attendance were discharged because they would not be in sympathy with the new régime. I learned afterwards that the specialist's boast failed, that so far as friends could see there was no change in the condition of the heart, that she grew weaker each day, and died ten days after I had been dismissed, or about four weeks after delivery.

Dr. Whittier did not consider the case as one of tachycardia due to exophthalmic goitre, or rather as a functional tachycardia in which exophthalmus had not developed. Physical examination showed a marked pallor; great distress in respiration; cold extremities; heart enlarged to right and to the left; apex outside the nipple line; no edema; rate 180 . I thought I heard a diastolic murmur at the base, but this diagnosis was not confirmed by a second sound heard in the femorals. This was July 22.

July 23 to July 24 Dr. Swift reported her as little more comfortable.

July 27 I saw her again. Respiration, 48; pulse, 148. In the right axillary region a pleural friction sound was heard, also fine râles. There was a marked pulsation of the veins in the neck, pulsation of the liver and a systolic murmur heard over the liver. These signs demonstrated a marked tricuspid regurgitation, evidently dependent upon sudden dilatation of the heart, as none of them were present five days previously. The question of a pulmonary embolism was raised in my mind, accounting for the sudden dyspnea. Dr. Swift's notes have shown how futile were the therapeutic suggestions we could make.

This was a case without septic infection, but one of acute and fatal dilatation without demonstrable cause, except the effect of labor upon a heart that previously was not absolutely sound, though no previous examination had suggested valvular disease.
TUBERCULIN IN TREATMENT OF PULMONARY TUBERCULOSIS:*

BY WALTER A. GRIFFin, M.D.,

Resident Physician, Sharon Sanatorium, Sharon, Mass.

TuBERculin has been used as a cure for tuberculosis since 1890 when Koch first gave it to the world. Few things have been received with such joy, and fortunately few things in life have created such disappointment. It is certain that the pendulum swung too far at first, - that hope for what this then new remedy would do was too great. And it is probable that it swung too far in the opposite direction when the hopes were so little realized.

Theoretically tuberculin should be of value as an immunizing agent. Experimentally it has immunized animals to a greater or less degree according to the observations of numerous investigators. Clinically its utility can still be called in question, for while some claim excellent results, others state that patients are made no whit better by its use. In general, however, those who have used it for the greatest length of time are most enthusiastic for continuing its use, while the investigator who has had only a few cases is more apt to give it up. There is practically no one who has used it to any extent, moreover, who would state that the injections work any injury when properly given; while even the most conservative are willing to say that probably fewer relapses occur in patients who have had the treatment.

Many varieties of tuberculin have been brought forward, some to be discarded and some to be used for years. The reason for the multiplicity of the products is that no one knows just what the toxin of the tubercle bacillus really is, and hence various methods of extraction have been employed to try to obtain the essential poison. The culture medium on which the bacilli grow certainly does not contain all of the toxin, while it does contain much that is needless or even harmful to the body into which it is injected. Koch's old tuberculin, since it consists of this medium, is not all that it should be and cannot do the good that a more refined product should be able to do. Koch's new tuberculin which contains the bacilli powdered as minutely as possible is not free from danger since it has been proven that virulent bacilli are present in it. These two products have been used most extensively up to the present time, although various individual workers have prepared products to which they have given special names, and for which they claim good results. Thus Klebs has brought out antiphthisin and tuberculocidin, Maragliano and von Ruck have prepared a watery extract, Beraneck has recently devised a new product, and within a year von Behring has announced that he has obtained a toxin by treating the bacilli in special ways which will effectively prevent the disease. These products are but samples of the various extractives from tubercle bacilli that have been brought forward * Read before the Plymouth District Medical Society, April 18,
1906 . 
from time to time. As yet no one can say that sure immunity is to be gained by employing any of them. At the same time it is reasonable to suppose that immunity may come from this direction of investigation. It is questionable if this immunity will ever be very strong, or at least strong enough so that animals may be made to produce a serum sufficiently powerful to passively immunize individuals to the same degree that diphtheria antitoxin immunizes.

Just what action tuberculin has upon the cells of the body is not definitely settled. Injected into healthy individuals it causes no inconvenience, but in the tuberculous it causes a reaction, possibly because it there meets certain cell derivatives which unite with it to make a distinct irritant. The original idea obtained that the cells in immediate proximity to the tubercular nidus were the sole ones acted upon, That these particular cells are stirred up, at least. with old tuberculin, is evident from the congestion which follows, as noted by increased signs in the lungs when the lesion is here, or by congestion about an infected larynx or an area of lupus. In addition the cells of the body generally are stimulated as is evident from the fact that progressively increased doses can be given without harm; and further that experimentally, at least, animals gain a slight immunity to infection by inoculation. This immunity is not so pronounced as when other products of the bacilli are used, or even avirulent bacilli themselves are injected, but up to the present time it has not been possible to grow bacilli from such a source or in such a way that one would feel free to use them on human beings. Thus Trudeau was able to immunize animals with cultures which had lost their virulence by long cultivation, but one would not dare to try to immunize men in the same way. Neither has it been possible to prepare bacilli known to be virulent in such a way that their use is unattended with risk. Attempts have been made in this direction and Koch's new tuberculin is the best known example of work along this line. Finally, as stated, numerous attempts have been made to extract the essential toxin from the bodies of the bacilli, and many of these extractives have been used clinically without, however, proving that they have any greater immunizing power than the old tuberculin. Nobody knows what the toxin is nor what particular extractive contains the most of it. Von Behring has heralded the latest product, and we certainly hope that he has found the sure and safe way to gain immunity.

When tuberculin was first introduced it was used indiscriminately upon incipient and faradvanced cases. Many patients were made tremendously sick, and some were undoubtedly hurried to their graves. To-day it is probable that practically none are injured even if none are helped. Far-advanced cases with fever are not now advised to take the drug although some of the bacillary extractives have been used in such cases by certain observers, and good results have been claimed. Neither are the large initial doses formerly employed now deemed permissible. Treatment is begun with but a fraction of a milligram, and the size of the dose is increased only so fast as may be without causing fever reaction. This slow method of procedure is commonly employed with all the tuberculins now in use. Months ensue before large doses can be given, and the whole course of the treatment may last a year.

As guides to determine how rapidly the injections may be pushed, reliance has to be put upon the temperature chart, and upon the general condition of the patient. A four-hour chart is commonly kept and if the temperature goes over $99^{\circ}$ the previous dose is repeated or even lessened. If any constitutional disturbance arises, as noted by headache or increased congestion of the tubercular focus, whether it be larynx or lung or any other part, the same caution as to dosage is employed. Recently Wright, of London, has devised a method of testing the power of the serum to increase phagocytosis. The process is called the determination of the opsonic index. Certain cases have their index increased by tuberculin, while others have it lowered. These latter, therefore, are not considered suitable cases for treatment. Furthermore, those cases which are at first suitable may show a lowering of the index if the drug is pushed too fast or given in too large doses. Practically, the technique to determine the index is a good deal involved and hardly to be undertaken outside of a well-equipped laboratory. One important thing in connection with the test, according to Wright, is that the opsonic index is raised to its highest point by small amounts of tuberculin, and he never reaches more than very moderate doses. In his work he uses Koch's new tuberculin. In other cases not controlled by the opsonic index it is the custom to increase the patient's power to receive the drug until he can withstand doses of 500 to $1,000 \mathrm{mgm}$. or even more ( $\frac{1}{2}$ to 1 cc. of crude tuberculin). It may be that in the future we shall consider these doses altogether too large. Certainly, it has been already established by clinical experience that there may be a point reached where the cells of the body will cease to be stimulated by tuberculin, and experimentally the same pathological changes are observed in the various organs of an animal killed with tuberculin as are observed after death from tuberculosis.

It is probable, therefore, that from evidence at hand patients will do as well or better by receiving rather moderate doses, and since after treatment has stopped for a while it cannot be renewed except by starting in with small doses it may be best, as indeed some advocate, to give more than one course of treatment, each lasting some months, and with months of intermission.

We have given tuberculin at the Sharon Sanatorium to three patients for a lengthy period during the last year and we have five at present under treatment. In all of these cases we have used Koch's old tuberculin, kindly supplied by the Saranac Lake Laboratory, except in two 
instances where von Ruck's watery extract was used for a while. In regard to this latter product, I found that the two patients who received it were in no wise made less susceptible to ordinary tuberculin, for after they had been worked up to $.650 \mathrm{gm}$. of the watery extract I gave them each $.01 \mathrm{gm}$. of tuberculin and caused a typical reaction. This does not mean necessarily that they had gained nothing in immunity, since the two products are so utterly different and since I had no way of testing the agglutinating power of their serum before and after the course of treatment, but it left a doubt as to the utility of using watery extract, and both patients are now receiving old tuberculin in small doses.

The method of procedure is as follows: fourhour charts are kept and on every other day the injections are given beginning with a quarter of a milligram, and increasing as rapidly as possible without causing a reaction. At first each increment is only a fraction of a milligram, but towards the end of a treatment a case can often take an increment of several centigrams without showing a reaction. It is not always possible to escape fever with some patients, the temperature frequently rising about a degree, but in this connection it is important to note that some claim greater improvement in those cases that have these mild reactions. Of the three cases discharged from the sanatorium two occasionally had a little fever during the treatment and these two were both discharged arrested, whereas the third case never showed any reaction but once, and went away still coughing and raising bacilli. The history of the three cases more in detail is as follows:

CASE 243. Admitted first April 20, 1903. There were well-marked signs all over the left side of the chest and at the top of the right. The sputa contained many tubercle bacilli. With ordinary sanatorium treatment the cough and expectoration ceased and the weight increased forty-two pounds. She was discharged at the end of eight months. June 11, 1904, she was readmitted for renewed cough and expectoration. For five months the physical signs and general condition remained about the same. Bacilli could be demonstrated in the sputa at any time. Finally, since there had been no appreciable change since entrance, Dr. Bowditch decided to try tuberculin. Twentyseven injections were given over a period of five months ranging from $.0005 \mathrm{gm}$. to $.600 \mathrm{gm}$. In all $2.1 \mathrm{gm}$. were given. Soon after the treatment was begun the cough increased, and at this time the bacilli were more numerous in the sputum in this case than in that of any other patient in the sanatorium; but this condition rapidly improved and for more than a month before discharge the cough and expectoration were nil, while the signs in the chest were likewise much improved, and I understand that she has remained in good general health since returning home.

CASE 342. This patient came with a history of a cough for five months. There were well-marked signs over the upper two thirds of the left chest, and tubercle bacilli were present in the sputum. Soon after the cough and expectoration increased and there was slight temperature in the afternoon. From this time there were numerous upsets and after the patient had been in the sanatorium three and a half months the signs had increased so as to be nearly all over the left side and with slight signs on the right. The voice was also husky and the larynx showed redness and swelling about the artynoids and redness of the vocal bands. At this time treatment was begun with tuberculin. At first there was a little fever after nearly every injection. The cough increased also and there were more bacilli in the sputum. Gradually all the signs and symptoms improved until at discharge there had been no cough or expectoration for some time and the signs in the chest had cleared up to a marked degree. Treatment was continued for a period of seven and one-half months. In all there were 58 injections, the last one being $.580 \mathrm{gm}$. By a recent report the cough and expectoration had not returned and the patient was feeling well.

CASE 337. This patient came with a history of cough for eight months. She showed well-marked signs in the upper left lung, and there were signs of beginning trouble in the right lung. Tubercle bacilli were numerous. The condition improved rapidly in the first three or four months, and then improvement came much more slowly, so that for two months or more there seemed to be no marked change in the condition, and the tubercle bacilli persisted in the sputum. Tuberculin treatment was begun about six months after entrance and was continued for seven months. The patient received in all 51 injections, the last one being $.700 \mathrm{gm}$. Only once was there a reaction following an increment of $.08 \mathrm{gm}$., toward the end of the treatment. The general condition improved somewhat, but the cough and expectoration remained about the same while bacilli persisted in the sputum. Since discharge this patient has had an attack of hemoptysis, but is in fair condition in another sanatorium.

These cases are striking since they would not be considered particularly favorable for tuberculin treatment, and since they were under observation for some months before treatment was instituted. This latter point is of especial importance since Dr. Bowditch has felt very strongly that the value of a treatment can be judged only after the patient has been under ordinary hygienic conditions for some time, otherwise the improvement which ought to be ascribed to sanitary living might be used in praise of a particular drug. In this small series of cases the evidence is indeed strong that tuberculin was of great value in at least two cases and of no harm in the third.

\section{Clinical glepartment.}

\section{DERMOID CYST WITH TWISTED PEDICLE AND TWISTED BROAD LIGAMENT.*}

By W. L. Burrage, M.d., Boston.

H. H., twenty-five years of age and mother of one child, entered the Gynecological Service of the Carney Hospital, Sept. 15, 1898. She had been married five years and besides a difficult labor four years ago had had an abortion at three months, three years before. In the past two years she had suffered with eight or ten attacks of severe pain in the right side.

Present attack of pain in the right side and loin has lasted for two weeks. Catamenia regular. Profuse vaginal discharge with bad odor. Dysuria.

Copper-colored eruption for two months on arms, * Read before the Boston Obstetrical Society, March 271906. 